\title{
(2) Effects of non-surgical treatment of chronic OPEN ACCESS periodontitis on insulin resistance and glucose tolerance in subjects without diabetes (PARODIA 2 study)
}

\author{
Arnel Redon Nana Nana (i) ,' Nadia-Flore Tsobgny Tsague, ${ }^{1}$ \\ Eric Lontchi-Yimagou (10, ${ }^{2,3}$ Charles Bengondo Messanga, ${ }_{1}^{1}$ Aurel Tankeu, ${ }^{4}$ \\ Jean-Claude Katte, ${ }^{5}$ Eric Balti Vounsia, ${ }^{4}$ Mesmin Dehayem, ${ }^{4}$ Eugene Sobngwi ${ }^{4,6}$
}

For numbered affiliations see end of article.

\section{Correspondence to} Dr Arnel Redon Nana Nana, Department of Periodontology, Oral and Maxillo-Facial Surgery, University of Yaounde I Faculty of Medicine and Biomedical Sciences, Yaounde, Cameroon; narnelredon@gmail.com Professor Eugene Sobngwi; sobngwieugene@yahoo.fr

Accepted 15 June 2021 Published Online First 14 July 2021

\section{ABSTRACT}

This study aimed to assess the effects of non-surgical periodontal treatment (NSPT) of chronic periodontitis on insulin sensitivity, glucose tolerance, and serum $C$ reactive protein (CRP) level in individuals without diabetes. Twenty individuals without diabetes with chronic periodontitis underwent NSPT, which consisted of complete scaling, polishing, root planing, and irrigation of the periodontal pockets with a 10\% povidone-iodine solution. Periodontal indices (plaque index, gingival bleeding index, pocket depth, and clinical attachment loss), insulin sensitivity using the Short Insulin Tolerance Test index (KITT), glucose tolerance derived from oral glucose tolerance test, and serum CRP level were measured before and 3 months after the intervention. This study was carried out at the National Obesity Center of Yaoundé Central Hospital, Cameroon. After 3 months, we observed significant improvement in periodontal parameters (all $\mathrm{p}<0.001)$ and insulin sensitivity $(3.72(2.99-4.17)$ $\% /$ min before treatment vs 4.04 (3.67-4.78) \%/ min after treatment, $p=0.001)$ and significant decrease in serum CRP level $(2.35(1.46-4.18) \mathrm{mg} / \mathrm{L}$ before vs $1.53(1.03-2.12) \mathrm{mg} / \mathrm{L}$ after, $\mathrm{p}=0.033)$. There was a trend toward improvement in glucose tolerance, although not statistically significant after the intervention. This study suggests that NSPT of chronic periodontitis in individuals without diabetes is associated with increased insulin sensitivity and decreased serum CRP levels.

Trial registration number NCT02830113.

\section{INTRODUCTION}

Diabetes is one of the most common metabolic disorders globally, and type 2 diabetes mellitus (T2DM) is considered an epidemic and accounts for approximately $90 \%$ of total cases. ${ }^{1}$ Insulin resistance plays a crucial part in the pathogenesis of T2DM and prospective studies have demonstrated that it is the best predictor of diabetes. ${ }^{2}$ Chronic inflammation is one of the factors that can induce insulin resistance. ${ }^{3}$ Proinflammatory cytokines have been demonstrated to induce insulin resistance. ${ }^{5}$ Insulin resistance can therefore contribute to the development of T2DM.

On the other hand, reducing proinflammatory cytokines has been shown to improve insulin resistance. ${ }^{67}$ Chronic periodontitis is responsible for a long-lasting inflammation of the tissue surrounding the tooth. It brings forth a systemic inflammation even though the inflammation, in this case, is localized to the periodontal tissues. ${ }^{89}$ Treatment of periodontitis results in a reduction of the inflammation not only at the periodontal level but also at the systemic level, and this is marked by a reduction in serum inflammatory markers. ${ }^{10}{ }^{11} \mathrm{C}$ reactive protein (CRP) has been considered a possible mediator of the relationship between periodontitis and systemic conditions. ${ }^{11}$ CRP levels increase in direct proportion to severity of periodontitis, ${ }^{12}$ and a decrease in periodontal inflammation results in the same proportional reduction in CRP levels. ${ }^{10} 11$

A possible reduction in severity of systemic diseases following periodontal therapy due to a decrease in inflammatory burden has been reported. ${ }^{13}{ }^{14}$ We hypothesized that non-surgical periodontal treatment (NSPT), including scaling, root planing, and subgingival irrigation with povidone-iodine, would improve insulin sensitivity by reducing inflammation. Therefore, this study aimed to evaluate the effects of a non-surgical treatment of chronic periodontitis on inflammation (by CRP serum level measurements), insulin sensitivity, and glucose tolerance in an adult Cameroonian population without diabetes.

\section{MATERIALS AND METHODS}

All subjects provided written informed consent.

\section{Setting and study population}

This study was conducted at the National Obesity Center of Yaoundé Central Hospital, Cameroon. This was a quasi-experimental, single-arm study where each subject was his/her 
own control. We enrolled 24 adult subjects with mild to severe chronic periodontitis according to the 2012 Centers for Disease Control and Prevention in partnership with the American Academy of Periodontology(CDC-AAP) classification. ${ }^{15}$ Exclusion criteria were a condition capable of influencing any of the study parameters (diabetes, hypertension, pregnancy, arthritis), any clinically diagnosed condition which is contraindicated for any of the procedures undertaken in the study (allergies, cardiopathies), patients who underwent any periodontal treatment within 6 months preceding the study, and patients with less than 20 vital teeth. Alcohol users, smokers, and subjects taking medications that may affect insulin resistance/sensitivity and/or inflammation before and during the study period were also excluded.

\section{Data collection}

We performed an interview and a complete physical examination on all subjects. We measured anthropometric parameters, insulin sensitivity, glucose tolerance, and serum CRP level before and 3 months after NSPT. Periodontal parameters, including O'Leary's plaque index (PI) ${ }^{16}$ Ainamo and Bay's gingival bleeding index (GBI), ${ }^{17}$ pocket depth (PD), and clinical attachment loss (CAL), were also measured before and 3 months after NSPT. Our primary endpoint measurement was insulin sensitivity and glucose tolerance, and our secondary outcome was improvement in the periodontal parameters.

\section{Anthropometric measurements}

We measured height to the nearest $0.5 \mathrm{~cm}$ and weight to the nearest $0.1 \mathrm{~kg}$ and calculated the body mass index (BMI) using Quetelet index in all subjects. ${ }^{18} \mathrm{We}$ measured waist and hip circumference to the nearest 0.5 $\mathrm{cm}$ and calculated the waist to hip ratio. Per cent body fat was measured by bio-impedancemetry (TANITA BC 420 MA, TANITA Corporation, Tabashi-ku, Tokyo, Japan).

\section{Periodontal examination}

PD and CAL using a periodontal probe (PCP UNC 12, $\mathrm{Hu}$-Friedy Manufacturing, Chicago, USA) were made on six sites (distobuccal, buccal, mesiobuccal, mesiolingual, lingual, and distolingual) of all teeth except the third molars. PD was measured as the distance from the free gingival margin (GM) to the bottom of the periodontal pocket.

CAL was measured as the distance from the cementoenamel junction (CEJ) to the bottom of the sulcus or the periodontal pocket: $\mathrm{CAL}=\mathrm{PD}-(\mathrm{CEJ}-\mathrm{GM})$, where GM was subject to recession and CEJ was exposed; the distance from the CEJ to the GM was given a negative value, and a positive value when the GM covered the CEJ.

O'Leary's PI was used to evaluate the quantity of dental plaque and Ainamo and Bay's GBI to evaluate gingival inflammation. Four sites (mesiobuccal, buccal, distobuccal, and lingual) were evaluated for each tooth except the third molars, and PI and GBI were expressed as the percentage of positive sites of the total number of examined sites.

\section{Metabolic assessments}

Insulin sensitivity measurement

Insulin sensitivity was evaluated using a short insulin tolerance test (SITT). For SITT, a fixed bolus of regular insulin dose of $0.15 \mathrm{IU} / \mathrm{kg}$ body weight was intravenously injected into the fasting subjects. Plasma glucose levels were measured every $3 \mathrm{~min}$ for $15 \mathrm{~min}$. The Short Insulin Tolerance Test Index (KITT) which represents the slope of the linear decline in plasma glucose was then calculated by dividing 0.693 by the plasma glucose half-life $(50 \%$ from baseline), denoted as $\mathrm{t}^{1 / 2}$.

\section{Glucose tolerance measurement}

Glucose tolerance was measured using the oral glucose tolerance test (OGTT). A 75 g OGTT was performed over $120 \mathrm{~min}$ after a 10 -hour overnight fast. Blood samples were collected from an antecubital vein before and $120 \mathrm{~min}$ after the $75 \mathrm{~g}$ oral glucose load for glucose determination. Impaired fasting glucose and impaired glucose tolerance were defined according to the definition of the American Diabetes Association.

\section{Biochemical measurements}

Immunoturbidimetric method with agglutination to latex was used for CRP dosing using a CRP turbidimetric CROMATEST dosage kit (Linear Chemicals, Barcelona, Spain). Plasma glucose was measured by the hexokinase method (Roche Diagnostics, Mannheim, Germany).

\section{Periodontal treatment}

Mechanical therapy was completed at a single unlimited time appointment. Patients were trained and given instructions on oral hygiene practices and received oral hygiene kits, after scaling, polishing, and root planing of all teeth were done. The scaling was performed with a magnetostrictive ultrasonic device (Denjoy DUS-1A, Changsha, China), followed by polishing of all teeth with a polishing paste. Root planing and periodontal pockets curettage under local anesthesia were done using Gracey curettes, followed by subgingival irrigation with a $10 \%$ povidone-iodine solution. Povidone-iodine has rapid anti-bacterial activity and resists deactivation by salivary or blood proteins. these are the properties that justify its use. After mechanical therapy, each individual received $0.12 \%$ chlorhexidine solution for daily mouthwash for 7 days, twice a day. Six weeks after the intervention, a complete periodontal reassessment was carried out with bacterial plaque control procedures, including scaling and polishing, and guidance on oral hygiene was repeated.

\section{Statistical analysis}

Statistical analysis was performed using Stata V.12.0 software. Continuous variables were expressed as median (IQR) and categorical variables as count (percentage). The Wilcoxon matched-pairs signed-rank test was used to compare variables before and 3 months after NSPT. A p value $\leq 0.05$ was considered statistically significant. 


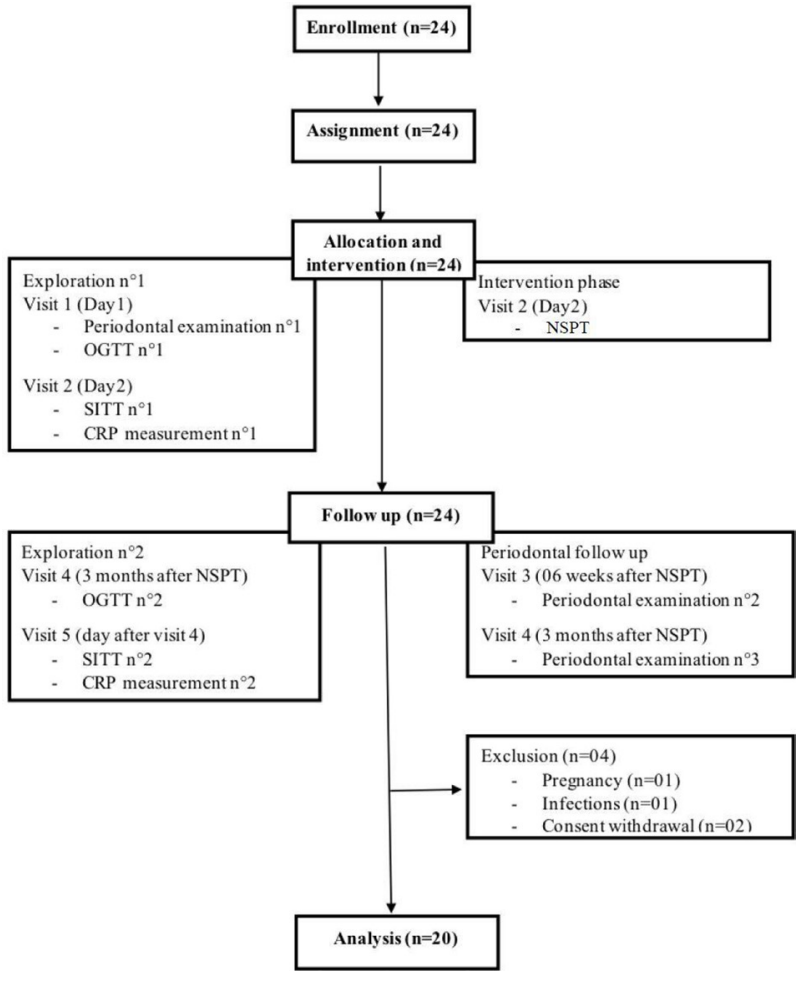

Figure 1 Diagram showing the flow of subjects through each stage of the trial. CRP, C reactive protein; OGTT, oral glucose tolerance test; SITT, short insulin tolerance test; NSPT, non-surgical periodontal treatment.

\section{RESULTS}

Twenty-four subjects were enrolled in this study. A total of 20 subjects completed the study after excluding four patients during the 3 months of follow-up. The study flow diagram is shown in figure 1 .

\section{Subject characteristics}

Women represented $25 \%$ of all patients (5 of 20). The age of the subjects ranged from 18 to 59 years, with a median of 37 (29-45) years. BMI ranged from 18.9 to $32.9 \mathrm{~kg} / \mathrm{m}^{2}$, with a median BMI of $23.4(21.35-26.57) \mathrm{kg} / \mathrm{m}^{2}$. According to the 2012 CDC-AAP classification, 8 subjects had severe periodontitis, 11 had moderate periodontitis, and 1 subject had mild periodontitis.

\section{Anthropometric parameters before and 3 months after NSPT}

BMI, waist and hip circumference, waist to hip ratio, and fat mass were not statistically different before and 3 months after NSPT (table 1).

\section{Periodontal parameters before and 3 months after treatment}

Three months after NSPT, we observed a statistically significant reduction in all periodontal parameters after 6 and 12 weeks (PI, GBI, PD, and CAL; all p<0.001; table 1).

\section{Metabolic parameters before and after 3 months of treatment}

As presented in table 1 , after 3 months, we observed a statistically significantly increase in KITT index (3.7 (3.04.2) $\% / \mathrm{min}$ before treatment vs $4.0(3.7-4.8) \% / \mathrm{min}$ after treatment, $\mathrm{p}=0.001$ ) and a decrease in serum CRP level (2.35 (1.46-4.18) $\mathrm{mg} / \mathrm{L}$ before vs $1.53(1.03-2.12) \mathrm{mg} / \mathrm{L}$ after, $\mathrm{p}=0.033)$. There was a trend toward improvement in glucose tolerance, although not statistically significant (median 116 (107-127) mg/dL before vs $112(99-126) \mathrm{mg} /$ $\mathrm{dL}$ after intervention, $\mathrm{p}=0.05$ ). Of the patients, $20 \%$ (4 of 20) had impaired glucose tolerance at baseline, and this proportion was reduced by half 3 months after NSPT.

Table 1 Anthropometric, periodontal, metabolic, and inflammatory parameters before and 3 months after NSPT

\begin{tabular}{lccc}
\hline Variables & Before NSPT & 3 months after NSPT & p value \\
\hline Anthropometric parameters & & & \\
\hline BMI $\left(\mathrm{kg} / \mathrm{m}^{2}\right)$ & $23.4(21.35-26.57)$ & $23.43(21.64-27.05)$ & 0.579 \\
\hline Hip circumference $(\mathrm{cm})$ & $97(92-100)$ & $98(94-110)$ & 0.321 \\
\hline Waist circumference $(\mathrm{cm})$ & $80(76-94)$ & $82(77-94)$ & 0.139 \\
\hline Waist to hip ratio & $0.84(0.79-0.87)$ & $0.83(0.79-0.87)$ & 0.262 \\
\hline Fat mass percentage (\%) & $14.75(10.77-28.12)$ & $17.9(12.12-27.32)$ & 0.118 \\
\hline Periodontal parameters & & & $<0.001$ \\
\hline Plaque index (\%) & $91.07(67.05-100)$ & $16.96(6.25-23.21)$ & $<0.001$ \\
\hline Gingival bleeding index (\%) & $62.8(40.44-78.1)$ & $1.08(0.00-4.50)$ & $<0.001$ \\
\hline Pocket depth (mm) & $3.12(2.98-3.45)$ & $1.6(1.52-1.81)$ & $<0.001$ \\
\hline Attachment loss (mm) & $3.35(3.08-4.0)$ & $1.7(1.57-2.05)$ & \\
\hline Metabolic and inflammatory parameters & & & 0.001 \\
\hline KITT (\%/min) & $3.72(2.99-4.17)$ & $4.04(3.67-4.78)$ & 0.023 \\
\hline FBG (mg/dL) & $88(83-94)$ & $85(79-89)$ & 0.050 \\
\hline 2-hour post-OGTT glycemia (mg/dL) & $116(107-127)$ & $112(99-126)$ & 0.033 \\
\hline CRP (mg/L) & $2.35(1.46-4.18)$ & $1.53(1.03-2.12)$ & \\
\hline
\end{tabular}

Data are median (IQR).

Significant $p$ values are in bold.

BMI, body mass index; CRP, C reactive protein; FBG, fasting blood glucose; KITT, glucose disappearance rate; NSPT, non-surgical periodontal treatment; OGTT, oral glucose tolerance test. 


\section{DISCUSSION}

In this pilot study, we investigated changes in metabolic, inflammatory, and periodontal parameters 3 months after NSPT with subgingival irrigation using povidone-iodine and mouth washing using chlorhexidine in individuals without diabetes with chronic periodontitis. The results showed that, 3 months after NSPT, there was a significant improvement in all studied periodontal parameters (PI, GBI, PD, and CAL), insulin sensitivity, and CRP level. Glucose tolerance improved by $7.44 \%$, but did not reach statistical significance. No significant changes in body fat percentage and BMI were observed.

Accumulating evidence has shown that treatment of periodontitis is associated with subsequent changes in the level of serum inflammatory markers. ${ }^{619-22}$ Our findings showed that treatment of periodontitis might contribute to improvement of insulin resistance likely via a reduction in inflammation. This study shows a trend toward improvement in glucose tolerance at 3 months post-NSPT as assessed by OGTT-derived parameter(s). We measured insulin sensitivity by SITT, a simple, easy, quick, low-cost, reproducible, and reliable method to evaluate insulin sensitivity in clinical practice and epidemiological studies. ${ }^{23}$ SITT is safe and has been shown to closely correlate with the hyperinsulinemic euglycemic clamp (gold standard) for insulin sensitivity measurement. ${ }^{24}$ This shift in insulin sensitivity may be associated with a reduction in inflammation as shown by a decrease in CRP, although the median CRP value was less than $5 \mathrm{mg} / \mathrm{mL}$ at the beginning of the study. The small sample size, the lack of a reference group, and the heterogeneous profile of the subjects remain the main limitations of this study. Nonetheless, body fat percentage, BMI, and waist to hip circumference ratio, which have known effects on insulin resistance, did not significantly change. Since each subject was his/her own control, the effect on insulin resistance of these parameters has therefore remained constant. Many studies suggest that periodontal disease, diabetes, and pre-diabetes share a common denominator, which is chronic inflammation characterized by an increased expression of common proinflammatory cytokines. However, the mechanisms between inflammation and these diseases are not yet fully understood. Since chronic periodontitis is an inflammatory disease, further randomized controlled trials controlling for confounders are needed to confirm its effect on blood glucose at the metabolic level.

\section{CONCLUSION}

To the best of our knowledge, this was the first study to evaluate the impact of non-surgical periodontal therapy on insulin resistance and glucose tolerance. Our results indicate that treating chronic periodontitis with NSPT reduces systemic inflammation and improves insulin sensitivity despite a marginal effect on glucose tolerance assessed using 2-hour postload glucose during OGTT in individuals without diabetes. Further, randomized controlled trials are needed to demonstrate the impact of NSPT on glucose tolerance and confirm its effect on insulin resistance.

\section{Author affiliations}

${ }^{1}$ Department of Periodontology, Oral and Maxillo-Facial Surgery, University of Yaounde I Faculty of Medicine and Biomedical Sciences, Yaounde, Cameroon
${ }^{2}$ Diabetes Research Center, Albert Einstein College of Medicine, Bronx, New York, USA

${ }^{3}$ Laboratory of Molecular Medicine and Metabolism, Biotechnology Center, Université de Yaoundé I, Yaounde, Cameroon

${ }^{4}$ Department of Internal Medicine and Specialties, University of Yaounde I Faculty of Medicine and Biomedical Sciences, Yaounde, Cameroon

${ }^{5}$ Department of Public Health, Faculty of Medicine and Biomedical Sciences, University of Yaounde, Yaounde, Cameroon

${ }^{6}$ National Obesity Center, Yaoundé Central Hospital, Yaoundé, Cameroon

Acknowledgements We are grateful to all the subjects of the study.

Contributors ARNN, MD, and ES designed the research. ARNN, N-FTT, and ES performed the research. ARNN, EL-Y, N-FTT, CBM, and ES contributed new reagents/analytic tools. ARNN, EL-Y, AT, JCK, and ES analyzed the data. ARNN, EL-Y, N-FTT, JCK, AT, and ES wrote the paper. ARNN, EL-Y, N-FTT, JCK, $\mathrm{CBM}, \mathrm{AT}, \mathrm{EB}, \mathrm{MD}$, and $\mathrm{ES}$ critically revised and adopted the manuscript. ES is the guarantor of this work. All authors approved the final version of the manuscript.

Funding The authors have not declared a specific grant for this research from any funding agency in the public, commercial or not-for-profit sectors.

Competing interests None declared.

Patient consent for publication Not required.

Ethics approval All procedures performed in studies involving human subjects were following the ethical standards of the institutional and/or national research committee and with the 1964 Helsinki Declaration and its later amendments or comparable ethical standards. The study received approval from the institutional and research ethical committee of the Faculty of Medicine and Biomedical Sciences and the institutional review board of the Yaoundé Central Hospital of Cameroon (no: AR/DHCY/CM/AD_V/L 26/01/2015).

Provenance and peer review Not commissioned; externally peer reviewed.

Open access This is an open access article distributed in accordance with the Creative Commons Attribution Non Commercial (CC BY-NC 4.0) license, which permits others to distribute, remix, adapt, build upon this work noncommercially, and license their derivative works on different terms, provided the original work is properly cited, an indication of whether changes were made, and the use is non-commercial. See: http://creativecommons.org/ licenses/by-nc/4.0/.

\section{ORCID iDs}

Arnel Redon Nana Nana http://orcid.org/0000-0002-2684-5138 Eric Lontchi-Yimagou http://orcid.org/0000-0003-1071-3154

\section{REFERENCES}

1 International Diabetes Federation. IDF diabetes atlas. 9th ed. Brussels: IDF, 2019.

2 Reaven GM. Role of insulin resistance in human disease (syndrome X): an expanded definition. Annu Rev Med 1993;44:121-31.

3 Shoelson SE, Lee J, Goldfine AB. Inflammation and insulin resistance. J Clin Invest 2006;116:1793-801.

4 Romeo GR, Lee J, Shoelson SE. Metabolic syndrome, insulin resistance, and roles of inflammation--mechanisms and therapeutic targets. Arterioscler Thromb Vasc Biol 2012;32:1771-6.

5 Fernandez CJ, George AS, Subrahmanyan NA, et al. Epidemiological link between obesity, type 2 diabetes mellitus and cancer. World I Methodol 2021:11:23-45

6 Mammen J, Vadakkekuttical RJ, George JM, et al. Effect of non-surgical periodontal therapy on insulin resistance in patients with type II diabetes mellitus and chronic periodontitis, as assessed by C-peptide and the homeostasis assessment index. J Investig Clin Dent 2017;8:e12221.

7 Nobles CJ, Mendola P, Mumford SL, et al. Metabolic syndrome and the effectiveness of low-dose aspirin on reproductive outcomes. Epidemiology 2019:30:573-81.

8 Nibali L, D'Aiuto F, Griffiths G, et al. Severe periodontitis is associated with systemic inflammation and a dysmetabolic status: a case-control study. I Clin Periodontol 2007:34:931-7.

9 Leira Y, Rodríguez-Yáñez M, Arias S, et al. Periodontitis is associated with systemic inflammation and vascular endothelial dysfunction in patients with lacunar infarct. J Periodontol 2019;90:465-74.

10 Kamil W, Al Habashneh R, Khader Y. Effects of non-surgical periodontal therapy on C-reactive protein and serum lipids in Jordanian adults with advanced periodontitis. J Periodont Res. oct 2011;46:616-21. 
11 Mohan M, Jhingran R, Bains VK, et al. Impact of scaling and root planing on C-reactive protein levels in gingival crevicular fluid and serum in chronic periodontitis patients with or without diabetes mellitus. J Periodontal Implant Sci 2014;44:158-68.

12 D'Aiuto F, Ready D, Tonetti MS. Periodontal disease and C-reactive proteinassociated cardiovascular risk. J Periodontal Res 2004;39:236-41.

$13 \mathrm{Du} \mathrm{Q}, \mathrm{Ma} X$. [Research progress of correlation between periodontal pathogens and systemic diseases]. Nan Fang Yi Ke Da Xue Xue Bao 2020;40:759-64.

14 Pradeep AR, Manjunath RGS, Kathariya R. Progressive periodontal disease has a simultaneous incremental elevation of gingival crevicular fluid and serum CRP levels. J Investig Clin Dent 2010;1:133-8.

15 Eke PI, Page RC, Wei L, et al. Update of the case definitions for populationbased surveillance of periodontitis. J Periodontol 2012;83:1449-54.

16 O'Leary TJ, Drake RB, Naylor JE. The plaque control record. J Periodontol 1972;43:38.

17 Ainamo J, Bay I. Problems and proposals for recording gingivitis and plaque. Int Dent J 1975;25:229-35.

18 Keys A, Fidanza F, Karvonen MJ, et al. Indices of relative weight and obesity. J Chronic Dis 1972;25:329-43.
19 Marcaccini AM, Meschiari CA, Sorgi CA, et al. Circulating interleukin-6 and high-sensitivity C-reactive protein decrease after periodontal therapy in otherwise healthy subjects. J Periodontol 2009;80:594-602.

20 Costa FO, Esteves Lima RP, Cortelli SC, et al. Effect of compliance during periodontal maintenance therapy on C-reactive protein levels: a 6 -year followup. J Clin Periodontol 2021;48:400-9.

21 Preshaw PM, Taylor JJ, Jaedicke KM, et al. Treatment of periodontitis reduces systemic inflammation in type 2 diabetes. J Clin Periodontol 2020;47:737-46.

22 Saraswathi IR, Sadasivan A, Koshi E, et al. Effect of nonsurgical periodontal therapy on serum level of interleukin- 6 and tumor necrosis factor- $\alpha$ in chronic periodontitis patients with and without hypothyroidism. J Contemp Dent Pract 2020;21:410-5.

23 Hirst S, Phillips DI, Vines SK, et al. Reproducibility of the short insulin tolerance test. Diabet Med 1993;10:839-42.

24 Bonora E, Moghetti P, Zancanaro C, et al. Estimates of in vivo insulin action in man: comparison of insulin tolerance tests with euglycemic and hyperglycemic glucose clamp studies. J Clin Endocrinol Metab $1989 ; 68: 374-8$ 